\title{
A hyperbolic diffusion model for stock prices*
}

\author{
Bo Martin Bibby ${ }^{1}$, Michael Sørensen ${ }^{2}$ \\ ${ }^{1}$ Department of Biometry and Informatics, Research Centre Foulum, P.O. Box 23, \\ DK-8830 Tjele, Denmark \\ ${ }^{2}$ Department of Theoretical Statistics, Institute of Mathematics, University of Aarhus, \\ DK-8000 Aarhus C, Denmark
}

\begin{abstract}
In the present paper we consider a model for stock prices which is a generalization of the model behind the Black-Scholes formula for pricing European call options. We model the log-price as a deterministic linear trend plus a diffusion process with drift zero and with a diffusion coefficient (volatility) which depends in a particular way on the instantaneous stock price. It is shown that the model possesses a number of properties encountered in empirical studies of stock prices. In particular the distribution of the adjusted log-price is hyperbolic rather than normal. The model is rather successfully fitted to two different stock price data sets. Finally, the question of option pricing based on our model is discussed and comparison to the Black-Scholes formula is made. The paper also introduces a simple general way of constructing a zero-drift diffusion with a given marginal distribution, by which other models that are potentially useful in mathematical finance can be developed.
\end{abstract}

Key words: Martingale estimating function, option pricing, quasi-likelihood, simulation, stochastic differential equation, volatility.

JEL classification: G12

Mathematics Subject Classification (1991): 62M05

\section{Introduction}

It is well documented that the distribution of stock returns typically has heavier tails than the normal distribution (see e.g. Mandelbrot 1963 and Fama 1965). In fact the distribution is often well fitted by a hyperbolic distribution, see Eberlein and Keller (1995) and Küchler et al. (1994). Moreover, the logarithm of the stock prices has in empirical studies turned out to have increments with a very low correlation. The increments are, however, not independent.

* Manuscript received: November 1995; final revision received: April 1996 
For instance, the squared increments show substantial correlation (see Fama 1965 and Taylor 1986, Chap. 2). Findings in this paper indicate that also the marginal distribution of this stochastic process can be fitted by a hyperbolic distribution.

Based on these empirical findings, we propose a diffusion process model for the logarithm of the stock price which, apart from a deterministic linear trend, is an ergodic process with hyperbolic invariant measure. This ergodic process is also a local martingale such that most trajectories will appear to have uncorrelated increments. Our model deviates only from the model behind the Black-Scholes formula by allowing the diffusion coefficient (volatility) corresponding to the log-price minus a deterministic linear trend to depend on the state of the process in a particular way.

In Sect. 2 we introduce the model and discuss some of its properties. Estimation of the parameters is discussed in Sect. 3 where data on the stock prices for two Danish companies are analysed. We use a martingale estimating function proposed by Bibby (1994), which generalizes earlier work by Bibby and Sørensen (1995a). We also check graphically how well the model fits the data. For this purpose we use residual plots as well as a non-parametric estimator of the diffusion coefficient. In Sect. 4 we briefly consider pricing of European call options and compare the result to the classical Black-Scholes formula.

\section{The model and its properties}

There is empirical evidence (Taylor 1986, Chap. 2) that the logarithm of the stock price is a process with nearly uncorrelated increments, but that the increments are not independent. This suggests a model for the stock price $S_{t}$ of the form

$$
S_{t}=\exp \left(\kappa t+X_{t}\right)
$$

where

$$
X_{t}=X_{0}+\int_{0}^{t} v\left(X_{s}\right) d W_{s} .
$$

By Itô's formula it follows that

$$
d S_{t}=S_{t}\left\{\left[\kappa+\frac{1}{2} v^{2}\left(\log S_{t}-\kappa t\right)\right] d t+v\left(\log S_{t}-\kappa t\right) d W_{t}\right\} .
$$

Note that if $v(x)$ is constant, then the model for $S_{t}$ is the geometric Brownian motion used in the derivation of the Black-Scholes formula. The process $X$ is a stochastically time-transformed Wiener process, so (2.1) is a simple and very natural generalization of the model used by Black and Scholes. The assumption that the drift of $\log S_{t}$ is a linear function of time, $\kappa t$, is not essential. It is made for the sake of simplicity and in order to change the geometric Brownian motion model as little as possible. Everything that is done in the rest of the paper can also be done when $\log S_{t}$ is assumed to have a general deterministic drift. 
By Theorem 2.2 in Engelbert and Schmidt (1985), the equation (2.2) with a given initial distribution has a weak solution if and only if the function $v^{-2}(x)$ is locally integrable. According to Theorem 3.2 in the same paper, the solution is unique in law if and only if $v^{2}(x)>0$ for all $x \in \mathbf{R}$. The scale measure of the solution has the density $s(x)=1$ with respect to the Lebesgue measure, and the speed measure has the density $m(x)=v^{-2}(x)$. For a definition and discussion of the scale measure and speed measure of a diffusion (see Karlin and Taylor 1981, Chap. 15). Hence

$$
\int_{0}^{\infty} s(x) d x=\int_{-\infty}^{0} s(x) d x=\infty,
$$

and if we choose $v(x)$ such that

$$
\int_{-\infty}^{\infty} v^{-2}(x) d x<\infty
$$

the diffusion process (2.2) is ergodic with invariant measure proportional to the speed measure, and $X_{t}$ converges in distribution to a probability measure with density proportional to $v^{-2}(x)$ (see e.g. Skorokhod 1989). Hence when $t$ is sufficiently large, the distribution of $X_{t}$ is well approximated by the distribution with density proportional to $v^{-2}(x)$. If $X_{0}$ has this distribution, $X_{t}$ will be stationary and exactly have density proportional to $v^{-2}(x)$ at all times.

Several interesting models can be obtained by choosing $v(x)$ appropriately, but we shall here concentrate on one particular choice and only briefly return to the general case at the end of this section. Empirical studies have shown that the distribution of the stock return, $\log S_{t+\Delta}-\log S_{t}$, is far from the normal distribution. In fact the tails of the distribution tend to be log-linear (when certain extreme events are excluded), see Eberlein and Keller (1995) and Küchler et al. (1994). Also the logarithm of the stock prices tends to have a distribution with log-linear tails, see the following section. A well-studied distribution with log-linear tails is the hyperbolic distribution introduced by Barndorff-Nielsen (1977). This distribution was used in the two papers just mentioned. The density of the hyperbolic distribution is proportional to

$$
\exp \left[-\alpha \sqrt{ } \delta^{2}+(x-\mu)^{2}+\beta(x-\mu)\right],
$$

where $x$ varies on the real line and the parameters satisfy the relations $\alpha>|\beta| \geqq 0, \delta>0$, and $\mu \in \mathbf{R}$. In order to obtain an ergodic diffusion with a hyperbolic invariant measure, we choose

$$
v^{2}(x)=\sigma^{2} \exp \left[\alpha \sqrt{ } \delta^{2}+(x-\mu)^{2}-\beta(x-\mu)\right] .
$$

With this choice the logarithm of the stock price will be approximately hyperbolically distributed after a sufficiently long time. It is hoped that also the increments, i.e. the stock price returns, are approximately hyperbolic. The distribution of increments over short intervals is a normal variance-mean mixture 


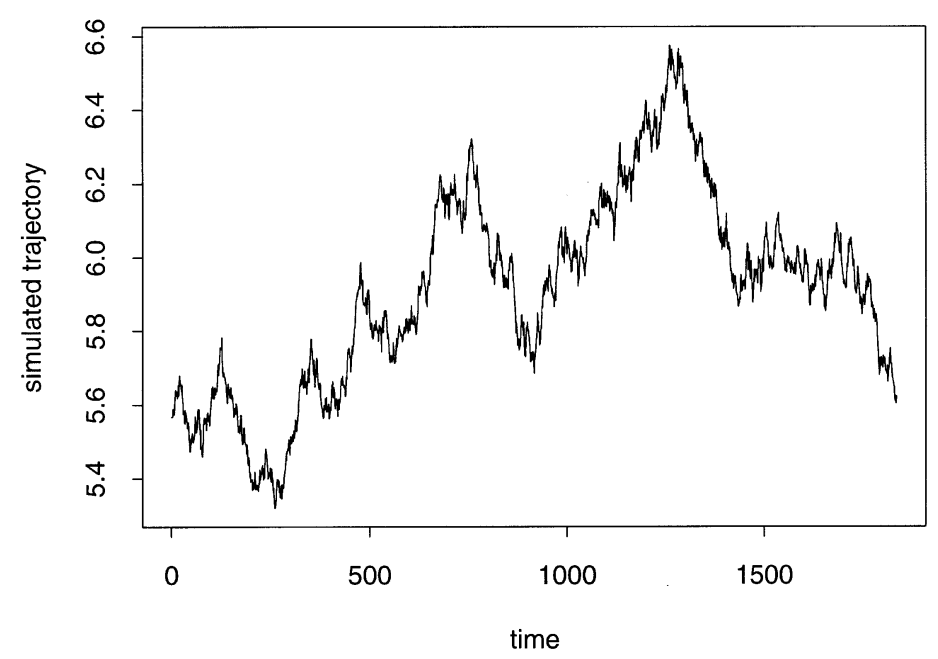

Fig. 1. A simulated trajectory of the diffusion process given by (2.2) and (2.4) with parameter values from the Baltica data in Sect. 3

and is hence likely to have heavy tails (see Barndorff-Nielsen et al. 1982). An increment over a long interval is the difference of two almost independent hyperbolic random variables, which has a distribution that is close to being hyperbolic. Note that with the specification (2.4) the volatility increases exponentially when the logarithm of the stock price deviates considerably from the trend given by $\kappa t$. It is not difficult to see that the boundaries $\pm \infty$ are entrance boundaries (see Karlin and Taylor 1981, p. 234).

In Fig. 1 one simulated sample path of the diffusion given by (2.2) and (2.4) is plotted. The time between consecutive simulated points is 1 and the parameter values are those obtained in Sect. 3 by fitting the model to the Baltica data (see Table 1). The simulation was done using the order 1.5 strong Taylor scheme (see Sect. 3).

In Fig. 2 and Fig. 3 histograms of the marginal distribution and of the distribution of the increments are given. These histograms were calculated from the sample path shown in Fig. 1 using the computer program HYP (see Blæsild and Sørensen 1992). All hyperbolic histograms in this paper are made using the HYP program. The marginal distribution shows considerable scatter; maybe because the single observations are far from independent. The increment distribution seems nicely hyperbolic. Here the scatter is less, probably because the observations are close to being uncorrelated.

The process $X_{t}$ given by (2.2) and (2.4) can, as mentioned above, be obtained by a stochastic time-transformation of a standard Wiener process. Specifically, let $B$ be a standard Wiener process, define $C_{t}$ by

$$
C_{t}=\int_{0}^{t} v^{-2}\left(B_{s}\right) d s,
$$




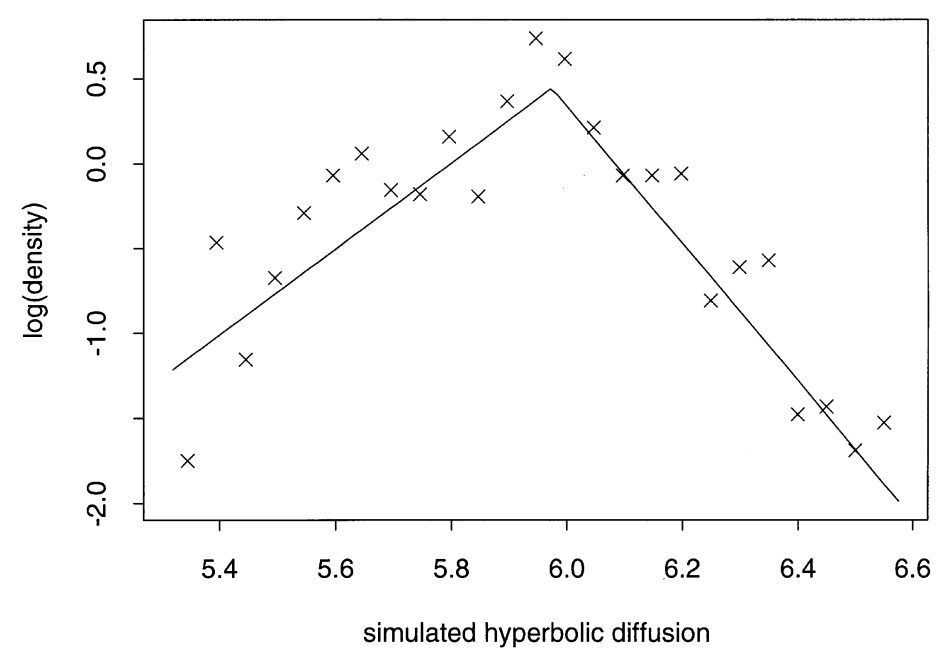

Fig. 2. The log histogram of the marginal distribution of the diffusion process given by (2.2) and (2.4) obtained from a simulated sample path. The full-drawn curve is a fitted hyperbolic density

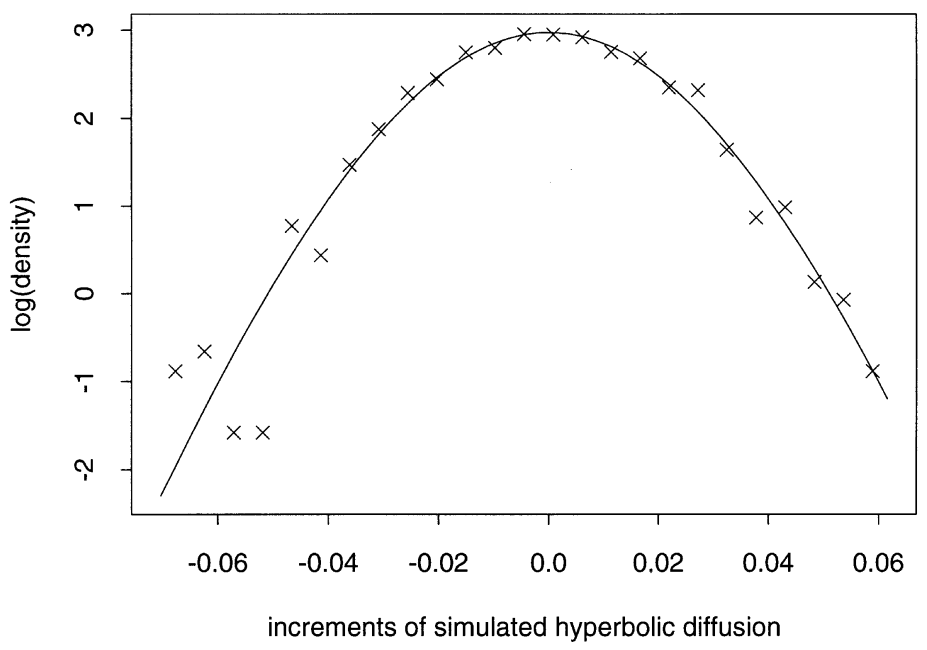

Fig. 3. The log histogram of the distribution of the increments of the diffusion process given by (2.2) and (2.4) obtained from a simulated sample path. The full-drawn curve is a fitted hyperbolic density

and let $T_{t}$ be the inverse of $C_{t}$, i.e.

$$
T_{t}=\inf \left\{s \geqq 0: C_{s}>t\right\} .
$$

Since $C_{t}$ is strictly increasing and $C_{\infty}=\infty$ almost surely (see Engelbert and Schmidt 1981), we see that $T_{t}$ defines a stochastic time-transformation. Ac- 
cording to Engelbert and Schmidt (1985)

$$
X_{t}=B_{T_{t}}
$$

is a weak solution of (2.2). We see that when the value of $B_{t}$ is in the centre of the hyperbolic distribution, $X_{t}$ behaves much like a Brownian motion. When $B_{t}$ is in the tail regions however, the process $T_{t}$ increases very rapidly. Thus $X_{t}$ will spend most of its time in the centre of the hyperbolic distribution even though $B_{t}$ will spend a lot of time in the tail regions. When $B_{t}$ is there, $X_{t}$ will vary much more rapidly than when $B_{t}$ is in the centre of the distribution.

A solution of (2.2) is a local martingale. With our specification (2.4) $X$ is, however, not a martingale. This can be seen from the following lemma.

Lemma 2.1 Suppose $M$ is a process with uncorrelated increments and that the first two moments of $M_{t}$ do not depend on $t$. Then either $M_{t}=M_{0}$ almost surely for all $t \geqq 0$ or $\operatorname{Var}\left(M_{t}\right)=\infty$.

Proof: Since $M$ has uncorrelated increments, $\operatorname{Var}\left(M_{t+s}\right)=\operatorname{Var}\left(M_{t+s}-M_{t}\right)+$ $\operatorname{Var}\left(M_{t}\right)$. Now $\operatorname{Var}\left(M_{t+s}\right)=\operatorname{Var}\left(M_{t}\right)<\infty$ implies $\operatorname{Var}\left(M_{t+s}-M_{t}\right)=0$.

In particular a martingale with a constant marginal distribution is either constant or has infinite variance. If the distribution of $X_{0}$ is the hyperbolic distribution with density proportional to $v^{-2}(x)$, the distribution of $X_{t}$ will be the same hyperbolic distribution for all $t$. Hence if $X$ was a martingale, the variance of $X_{t}$ should, according to Lemma 2.1, be infinite, but the hyperbolic distribution has finite variance. Note that $X$ is a new example of a uniformly integrable local martingale which is not a martingale.

The sample path of $X$ will, in spite of this, typically behave as if $X$ had uncorrelated increments. This is because $X$ is a local martingale, i.e. there exists an increasing sequence of stopping times $\tau_{n}$ such that $\tau_{n} \rightarrow \infty$ and such that $X_{t \wedge \tau_{n}}$ is a martingale for every $n \geqq 1$. Up to the stopping time $\tau_{n}$ the process $X_{t}$ has uncorrelated increments. Thus a finite discrete-time sample from $X_{t}$ will typically appear to have uncorrelated increments.

There is no doubt that also processes defined by (2.2) with $v^{2}(x)$ proportional to the inverse of other density functions than the hyperbolic density will turn out to be useful as models in mathematical finance. As long as $v^{2}(x)$ is chosen proportional to the inverse of a density with finite second moment, Lemma 2.1 shows that the corresponding diffusion is not a martingale; only a local martingale. However, if $v^{2}(x)$ is proportional to the inverse of a density function with infinite first moment, i.e. if

$$
\int_{-\infty}^{\infty}|x| v^{-2}(x) d x=\infty
$$

then the boundaries $\pm \infty$ are natural boundaries for the solution $X$ to (2.2) (see Karlin and Taylor 1981, p. 234). This implies, by Theorem 3 in Arbib (1965), that $X$ is a martingale. We see that, for instance, the choice $v^{2}(x)=1+x^{2}$ implies that $X$ is a martingale with Cauchy distributed marginals. 
It might seem preferable to model the stock price process by

$$
d S_{t}=S_{t}\left(\kappa+X_{t}\right) d t
$$

where $X$ solves (2.2) or is some other diffusion process with hyperbolic marginals. However, such a model would imply a stock price process with bounded variation on finite intervals so that there would be a possibility of arbitrage in the continuous-time option pricing theory (see Harrison and Pliska 1981). It might be argued that one should not take an arbitrage possibility based on the infinitesimal structure of a model too seriously, since the structure at this level does probably not correspond to anything real. However, we can avoid any such criticism by modelling the stock price process by $(2.3)$ and (2.4).

\section{Data and estimation}

In this section we consider some empirical data. We have looked at the daily stock price for Baltica, which is a Danish insurance company, and Aarhus Oliefabrik, a large producer of cooking oils. The time-period is from January 3rd, 1983 to April 30th, 1990. The time-unit is trading days, i.e. weekends are ignored so that Monday follows Friday. We only consider the stock prices on trading days where Baltica shares and Aarhus Oliefabrik shares respectively have been traded. Therefore there can be more than one day between two observations.

If we denote the price of a particular stock at time $t>0$ by $S_{t}$, then the observations are $S_{t_{1}}, \ldots, S_{t_{n}}$, where $t_{1}, \ldots, t_{n}$ are the trading days of the stock. We consider the log-price of a stock adjusted for trend, that is

$$
X_{t_{i}}=\log S_{t_{i}}-\kappa t_{i}, \quad i=1, \ldots, n .
$$

For each stock the slope $\kappa$ is determined by ordinary linear regression. For the Baltica shares we found the value $\kappa=0.00101$, and for the Aarhus Oliefabrik shares $\kappa=0.00033$. Figure 4 shows the adjusted log-price of the Baltica shares.

We want to model the adjusted log-price of the shares considered here using the hyperbolic diffusion process defined as the solution of

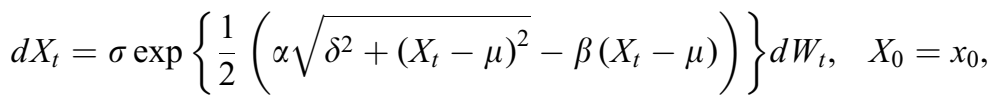

see (2.2) and (2.4). We saw from the simulations of the hyperbolic diffusion that we would expect the marginal density to be close to a hyperbolic density and the marginal density of the increments of the process to be closer to a hyperbolic density if the hyperbolic diffusion model is reasonable to describe the adjusted log-price. Figures 5 and 6 show the log-histograms for the adjusted log-price and the increments of the adjusted log-price respectively in the case of the Baltica shares. 


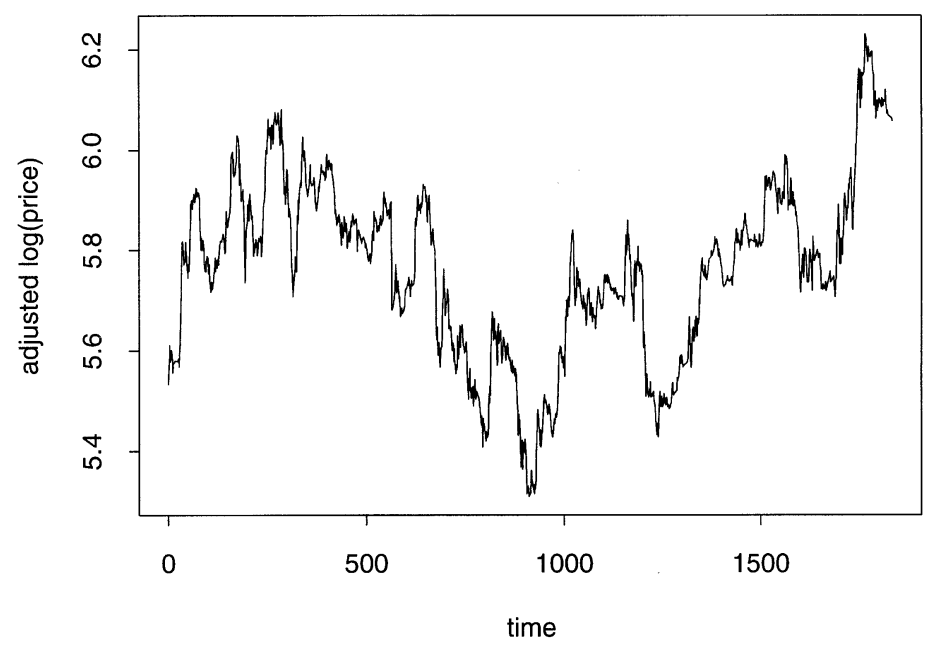

Fig. 4. The trend adjusted log price of the Baltica shares plotted against time

We see from Figs. 5 and 6 that the adjusted log-price of Baltica shares has a density close to a hyperbolic density and that the increments of the adjusted log-price of Baltica shares has a density that is closer to a hyperbolic density. This is what we would expect if the hyperbolic diffusion model is appropriate.

Several diffusion processes have a hyperbolic stationary distribution. One other example can be found in Küchler et al. (1994). The hyperbolic diffusion process given by the solution of (3.1) is the only one, though, without a drift. In order to check whether the shape of the diffusion coefficient for the hyperbolic diffusion appropriately accounts for the variability in the data studied in this section, we considered the non-parametric kernel estimator of the squared diffusion coefficient $v^{2}(x)$ proposed in Florens-Zmirou (1993). This estimator is a local quadratic variation estimator of the squared diffusion coefficient and is given by

$$
V_{n}(x)=\frac{\sum_{i=1}^{n} 1_{\left\{\left|X_{t_{i-1}}-x\right|<h\right\}}\left(X_{t_{i}}-X_{t_{i-1}}\right)^{2}}{\sum_{i=1}^{n} 1_{\left\{\left|X_{t_{i-1}}-x\right|<h\right\}}\left(t_{i}-t_{i-1}\right)},
$$

where $h$ is the bandwidth to be chosen appropriately.

Figure 7 shows the non-parametric estimator of the squared diffusion coefficient and the squared diffusion coefficient corresponding to the hyperbolic diffusion process given by (3.1) for a suitable choice of the parameters in the case of the Baltica shares. The parameter values used to make the plot of the squared diffusion coefficient in Fig. 7 are the ones found by the estimation method explained below (see Table 1). We see from Fig. 7 that for a bandwidth value of $h=0.2$ the non-parametric estimate of the squared diffusion 


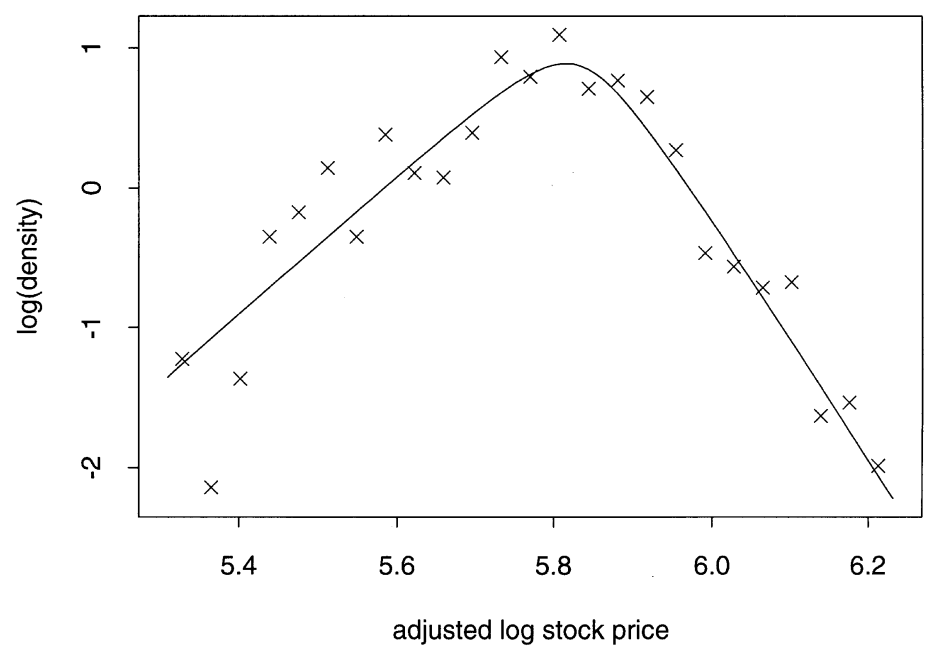

Fig. 5. The log-histogram for the adjusted log-price of the Baltica shares. The full drawn curve is a fitted hyperbolic density

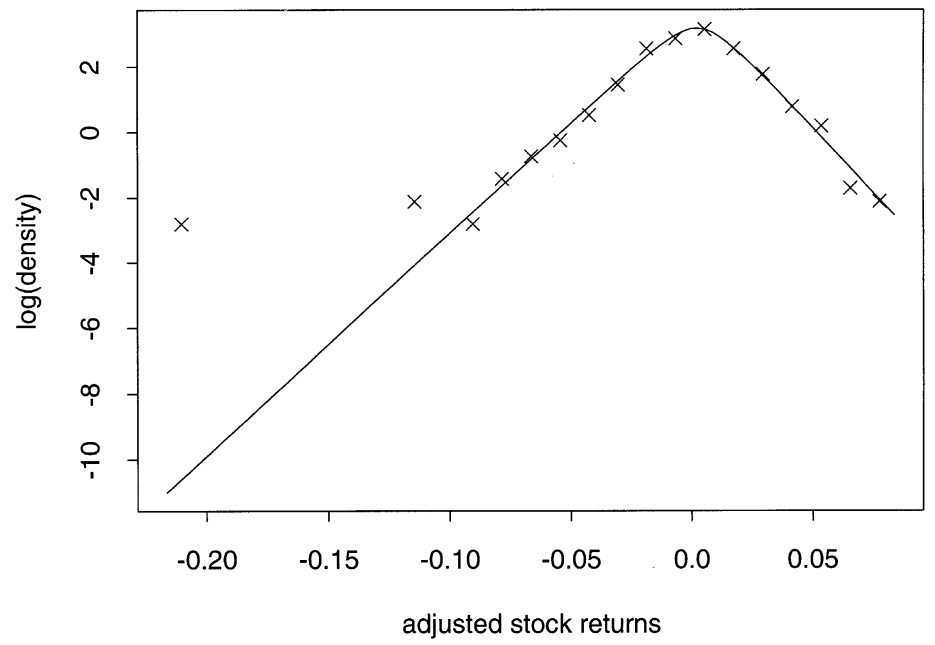

Fig. 6. The log-histogram for the increments of the adjusted log-price of the Baltica shares. The full-drawn curve is a fitted hyperbolic density

coefficient is close to the parametric estimate based on the hyperbolic diffusion process.

Even though the stationary distribution of the hyperbolic diffusion process given as the solution of (3.1) is known to be hyperbolic, the transition density is unknown. Therefore, inference about the parameters entering (3.1) cannot be based on the likelihood function. In Bibby and Sørensen (1995a) it is proposed that in such cases inference could be based on the martingale estimating function emerging by compensating the discretized continuous-time score function to make it a martingale. When a part of the parameter of interest only enters 


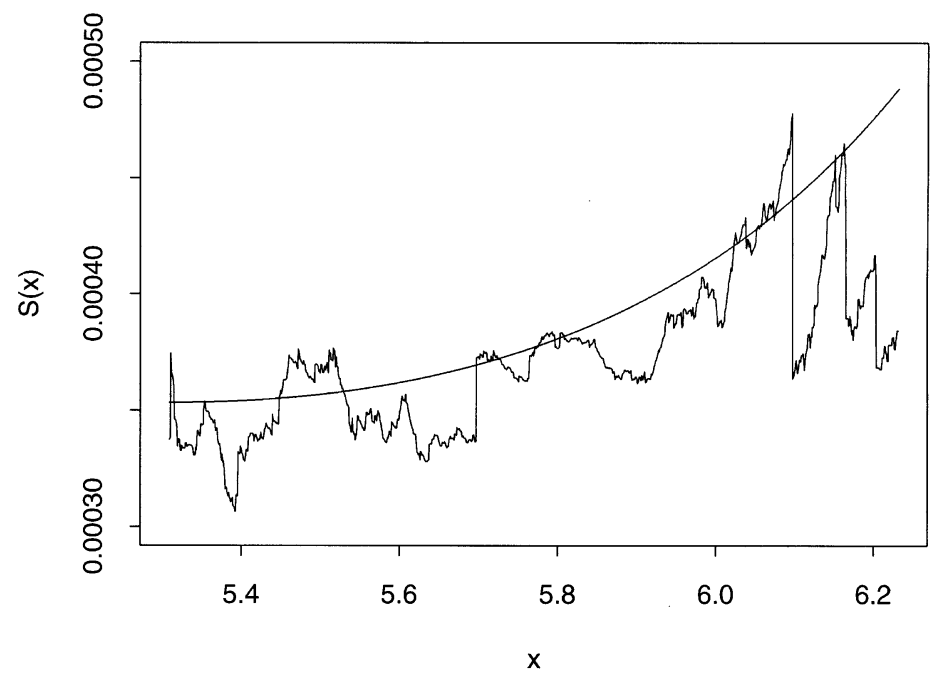

Fig. 7. The non-parametric estimator $V_{n}(x)$ (the jagged curve) of the squared diffusion coefficient $(h=0.2)$ and the squared diffusion coefficient (the smooth curve) corresponding to the hyperbolic diffusion. Both curves are estimated from the Baltica share data

the diffusion coefficient, the approach of Bibby and Sørensen (1995a) cannot be applied right away. In Bibby (1994) martingale estimating functions of the form

$$
\begin{aligned}
K_{n}(\theta)=\sum_{i=1}^{n}[ & g_{i}\left(X_{t_{i-1}} ; \theta\right)\left(X_{t_{i}}-F_{i}\left(X_{t_{i-1}} ; \theta\right)\right) \\
& \left.+h_{i}\left(X_{t_{i-1}} ; \theta\right)\left(\left(X_{t_{i}}-F_{i}\left(X_{t_{i-1}} ; \theta\right)\right)^{2}-\phi_{i}\left(X_{t_{i-1}} ; \theta\right)\right)\right],
\end{aligned}
$$

were considered. Here $F_{i}$ is the conditional mean and $\phi_{i}$ is the conditional variance of $X_{t_{i}}$ given $X_{t_{i-1}}$, and $\theta$ is a vector of model parameters. The functions $g_{i}$ and $h_{i}$ are of the same dimension as $\theta$. For ergodic diffusion processes, subject to mild regularity conditions on the functions $g$ and $h$, it was shown in Bibby (1994) that as the number of observations tends to infinity there exists with a probability tending to one - a solution of the equation $K_{n}(\theta)=0$ which is a consistent estimate of $\theta$ and which is asymptotically normally distributed.

As $X_{t}$ is a local martingale, there cannot be much information about the parameters in $F$, so we will use a martingale estimating function with $F_{i}(x ; \theta)$ replaced by $x$ and with $\phi_{i}$ replaced by the conditional expectation $\tilde{\phi}_{i}$ of $\left(X_{t_{i}}-\right.$ $\left.X_{t_{i-1}}\right)^{2}$ given $X_{t_{i-1}}$. Thus unnecessary numerical inaccuracies in the calculation of $F$ are avoided.

For small $\Delta_{i}=t_{i}-t_{i-1}$ an approximation to the optimal estimating function - optimal in the sense of minimizing a distance to the unknown score function (Godambe and Heyde 1987) - is given by

$$
\tilde{K}_{n}(\theta)=\sum_{i=1}^{n} \frac{\dot{v}\left(X_{t_{i-1}} ; \theta\right)}{\left(t_{i}-t_{i-1}\right) v\left(X_{t_{i-1}} ; \theta\right)^{3}}\left(\left(X_{t_{i}}-X_{t_{i-1}}\right)^{2}-\tilde{\phi}_{i}\left(X_{t_{i-1}} ; \theta\right)\right),
$$


where $v$ is the diffusion coefficient and a dot denotes differentiation with respect to $\theta$ (see Bibby and Sørensen 1995b). We will use the martingale estimating function defined by (3.2) to make inference about the parameter $\theta$. In this case $\theta$ denotes the vector $(\alpha, \beta, \delta, \mu, \sigma)^{T}$.

In practice the conditional expectation $\tilde{\phi}$ has to be calculated using simulations. More specifically $\tilde{\phi}\left(X_{t_{i-1}} ; \theta\right)$ is replaced by

$$
\frac{1}{N} \sum_{j=1}^{N}\left(X_{t_{i}}^{(j)}-X_{t_{i-1}}\right)^{2}, \quad i=1, \ldots, n,
$$

where $X_{t_{i}}^{(j)}$ is the $j^{\prime}$ th realization of $X_{t_{i}}$ according to, for instance, the strong Taylor scheme of order 1.5 (see Kloeden and Platen 1992, p. 351). The scheme is given by

$$
\begin{aligned}
X_{t_{i-1}+\frac{\Delta_{i} k}{m}}= & X_{t_{i-1}+\frac{\Delta_{i}(k-1)}{m}}+v_{k-1} \Delta W+\frac{1}{2} v_{k-1} v_{k-1}^{\prime}\left\{(\Delta W)^{2}-\frac{1}{m}\right\} \\
& +\frac{1}{2} v_{k-1}^{2} v_{k-1}^{\prime \prime}\left\{\Delta W \frac{1}{m}-\Delta Z\right\} \\
& +\frac{1}{2} v_{k-1}\left(v_{k-1} v_{k-1}^{\prime \prime}+\left(v_{k-1}^{\prime}\right)^{2}\right)\left\{\frac{1}{3}(\Delta W)^{2}-\frac{1}{m}\right\} \Delta W,
\end{aligned}
$$

where

$$
v_{k-1}=v\left(X_{t_{i-1}+\frac{\Delta_{i}(k-1)}{m}} ; \theta\right), \quad k=1, \ldots, m,
$$

and where $v_{k-1}^{\prime}$ and $v_{k-1}^{\prime \prime}$ are defined similarly. A prime denotes differentiation with respect to $x$. Moreover, $\sqrt{ } m \Delta W=U_{1}, 2 m^{3 / 2} \Delta Z=\left(U_{1}+U_{2} / \sqrt{ } 3\right)$, and $U_{1}$ and $U_{2}$ are independent $N(0,1)$-distributed random variables.

Table 1. The parameter estimates for Baltica and Aarhus Oliefabrik based on the martingale estimating function $\tilde{K}$

\begin{tabular}{lrcccc} 
& $\alpha$ & $\beta$ & $\delta$ & $\mu$ & $\sigma$ \\
\hline Baltica & 4.4875 & -3.8412 & 1.1949 & 7.2915 & 0.0047 \\
Aarhus Oliefabrik 6.8396 & -1.1496 & 1.1840 & 5.7512 & 0.0004
\end{tabular}

In the estimation procedure the number of repititions was fixed at $N=25$. The number of points between consecutive observation times was fixed at $m=$ 25. The result of the estimation is given in Table 1.

In order to validate the hyperbolic diffusion model in connection with the stock price data here we calculate the uniform residuals considered by Pedersen (1994), that is

$$
U_{i}(\theta)=F\left(t_{i-1}, X_{t_{i-1}}, t_{i}, X_{t_{i}} ; \theta\right), \quad i=1, \ldots, n
$$

where $F$ is the transition distribution function given by

$$
F(s, x, t, y ; \theta)=P_{\theta}\left(X_{t} \leqq y \mid X_{s}=x\right) \quad s<t, \quad x, y \in \mathbf{R} .
$$




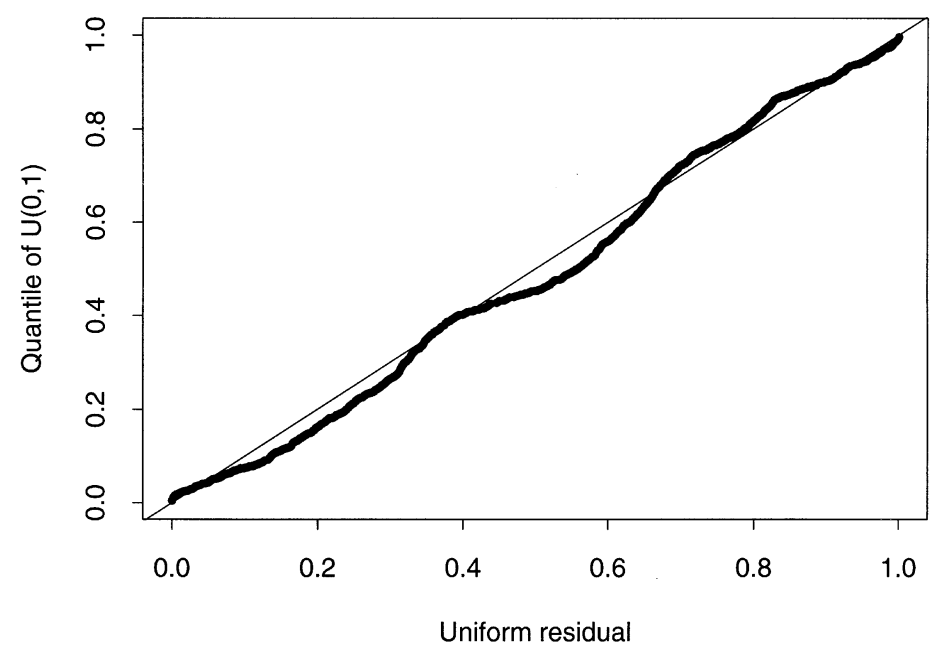

Fig. 8. A uniform quantile plot of the residuals $U_{i}$ corresponding to the hyperbolic diffusion model for the Baltica stock price data. The line is the unit line

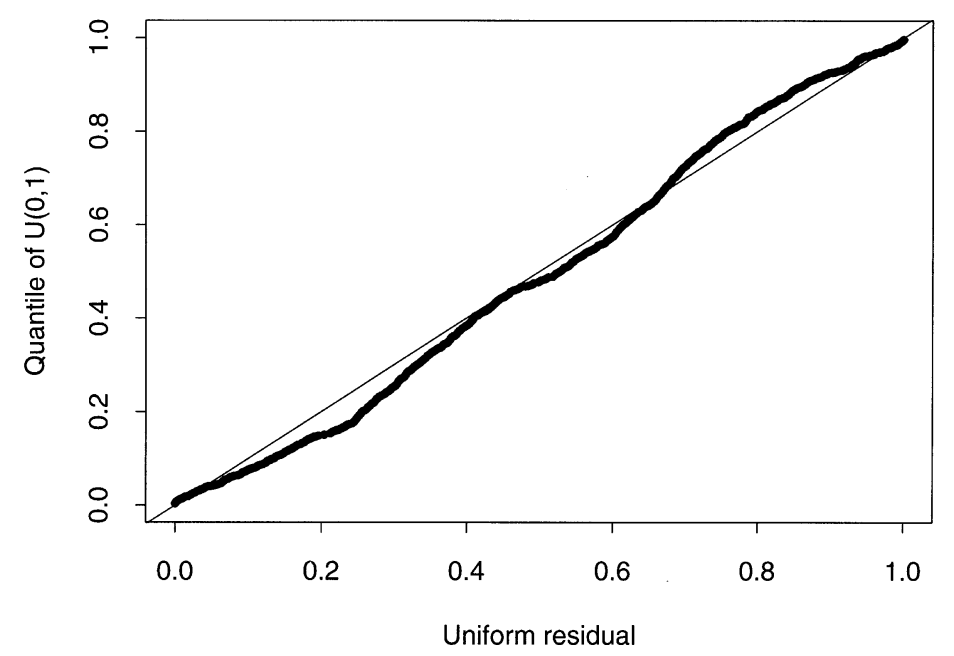

Fig. 9. A uniform quantile plot of the residuals $U_{i}$ corresponding to the hyperbolic diffusion model for the Aarhus Oliefabrik stock price data. The line is the unit line

Of course the transition distribution function is unknown and so the uniform residuals have to be calculated using simulations like for the conditional variance. If the hyperbolic diffusion model is the true model for the stock price data then the uniform residuals are independent and uniformly distributed on the unit interval. Figures 7 and 8 show plots of the uniform residuals against the quantiles of the uniform distribution on the unit interval for Baltica and Aarhus Oliefabrik. 


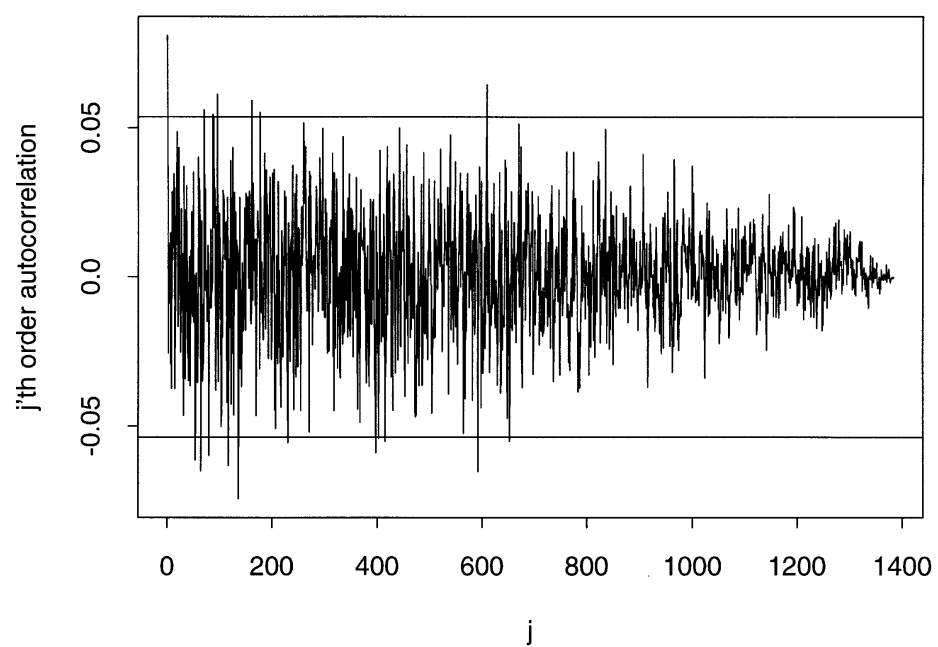

Fig. 10. The estimated $j$ 'th autocorrelation for the uniform residuals corresponding to the Baltica data as a function of $\mathrm{j}$

The plots in Figs. 8 and 9 support that the hyperbolic diffusion model (3.1) is a rather good model for the stock price data considered here. A test statistic based on the uniform residuals is given by

$$
X^{2}=-2 \sum_{i=1}^{n} \log U_{i}(\theta)
$$

If the uniform residuals are independent and uniformly distributed on the unit interval, which is the case if the model is true, we have that $X^{2} \sim \chi^{2}(2 n)$. For the Baltica shares $X^{2}=2722.061$, and for the Aarhus Oliefabrik shares $X^{2}=$ 2521.559. With $n=1385$ for the Baltica data and $n=1305$ for the Aarhus Oliefabrik data neither value of the test statistic give reason to rejecting that the uniform residuals are independent and uniformly distributed. Figure 10 shows the estimated autocorrelation function for the uniform residuals corresponding to the Baltica shares. The confidence bounds are given by $\pm 2 / \sqrt{ } n$ that would be appropriate if the uniform residuals were independent and normal. Based on Fig. 10 we conclude that the uniform residuals appear to be uncorrelated. The same plot for the Aarhus Oliefabrik shares shows a similar pattern in the estimated autocorrelation function for the residuals.

\section{Option pricing}

We consider a financial market consisting of a bond with fixed interest rate $r$ and a stock, the price process $S_{t}$ of which is given by (2.3) and (2.4). The discounted price process $\tilde{S}_{t}=e^{-r t} S_{t}$ solves the equation

$d \tilde{S}_{t}=\tilde{S}_{t}\left\{\left[\kappa-r+\frac{1}{2} v^{2}\left(\log \tilde{S}_{t}-(\kappa-r) t\right)\right] d t+v\left(\log \tilde{S}_{t}-(\kappa-r) t\right) d W_{t}\right\}$. 
Since

$$
\int_{0}^{t} \xi_{s}^{2} d s<\infty
$$

$P$-almost surely, where

$$
\xi_{s}=\frac{\kappa-r+{ }_{2}^{1} v^{2}\left(\log \tilde{S}_{s}-(\kappa-r) s\right)}{v\left(\log \tilde{S}_{s}-(\kappa-r) s\right)},
$$

it follows from Theorem 3.1 in Hansen (1995) that the market considered here is complete in the following sense. Define

$$
N_{t}=\exp \left\{-\int_{0}^{t} \xi_{s} d W_{s}-\frac{1}{2} \int_{0}^{t} \xi_{s}^{2} d s\right\}
$$

Then any contingent claim $H$ depending on the values of $S_{t}$ for $t \leqq T$ and satisfying $E\left(H N_{T}\right)<\infty$ can be hedged by an admissible self-financing trading strategy. The fair price of $H$, defined as the minimum initial capital needed to hedge $H$, is given by

$$
\pi(H)=E\left(H N_{T}\right) .
$$

The process $N$ is the Dolean-Dade exponential of the local martingale $-\int_{0}^{t} \xi_{s}$ $d W_{s}$ and is hence itself a local martingale. Since a non-negative local martingale is a supermartingale (by Fatou's Lemma), we see that $E\left(N_{t}\right) \leqq 1$, for all $t \geqq 0$. We assume that our probability space is rich enough that a probability measure $\tilde{P}$ exists under which $\tilde{S}$ solves the stochastic differential equation

$$
d Z_{t}=Z_{t} v\left(\log Z_{t}-(\kappa-r) t\right) d \tilde{W}_{t}
$$

where $\tilde{W}$ is a Wiener process under $\tilde{P}$. Under $\tilde{P}$ the process $Z_{t}^{*}=\exp ((r-\kappa) t)$ $Z_{t}$ solves the equation

$$
d Z_{t}^{*}=Z_{t}^{*}\left\{(r-\kappa) d t+v\left(\log Z_{t}^{*}\right) d \tilde{W}_{t}\right\},
$$

and for $Z^{*}$ the boundary zero of the state-space is attainable. It is, more precisely, a regular boundary if $\alpha+\beta>1$ and an exit boundary if $\alpha+\beta \leqq 1$. It follows that $Z_{t}^{*}$ can hit the boundary zero before the maturity time $T$ with a positive probability. Since $\exp (-|\kappa-r| T) \leqq \exp ((\kappa-r) t) \leqq \exp (|\kappa-r| T)$ for $t \in[0, T]$, the same is true of the solution to (4.4). From these considerations it follows that $\tilde{P}$ cannot be equivalent to $P$. However, by Theorem 2.1 in Rydberg (1996) the measures $\tilde{P}$ and $P$ are equivalent on the $\sigma$-field of events before the stopping time $\tau_{n}=\inf \left\{t: Z_{t} \leqq n^{-1}\right\}$ for every $n \geqq 1$. They are therefore also equivalent up to $T \wedge \tau_{n}$, and the Radon-Nikodym derivative is $N_{T \wedge \tau_{n}}$. Hence for all $n \geqq 1$ the fair price of $H$ is

$$
\begin{aligned}
\pi(H) & =E\left(1_{\left\{\tau_{n} \leqq T\right\}} H N_{T}\right)+E\left(1_{\left\{\tau_{n}>T\right\}} H N_{T}\right) \\
& =E\left(1_{\left\{\tau_{n} \leqq T\right\}} H N_{T}\right)+\tilde{E}\left(1_{\left\{\tau_{n}>T\right\}} H\right),
\end{aligned}
$$




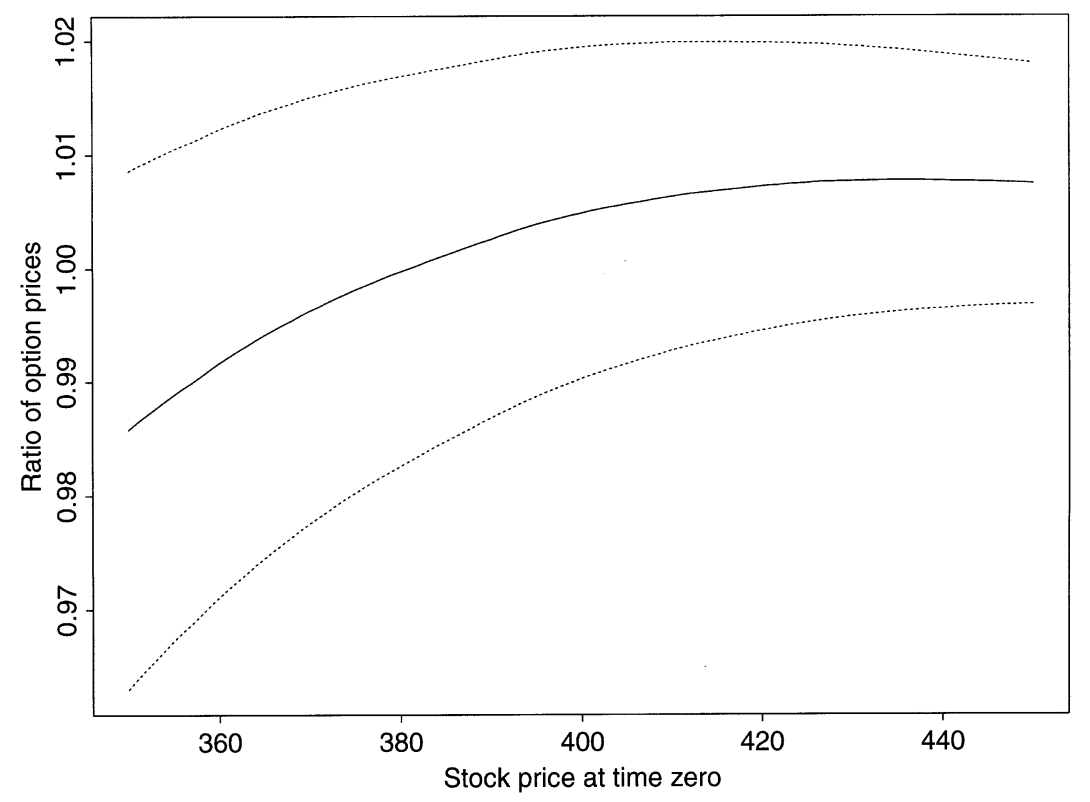

Fig. 11. The ratio of the option price (4.5) obtained using the hyperbolic diffusion model to the option price using the Black-Scholes model as a function of the stock price at time zero. The dotted curves are plus-minus twice the sample standard error

where $\tilde{E}$ denotes expectation under $\tilde{P}$. By the monotone convergence theorem

$$
\pi(H)=\tilde{E}\left(1_{\{\tau>T\}} H\right),
$$

where $\tau=\lim _{n \rightarrow \infty} \tau_{n}=\inf \left\{t: Z_{t}=0\right\}$. Here we have used that a solution of (4.1) is strictly positive such that $P(\tau \leqq T)=0$. Note that $E\left(N_{T}\right)=\tilde{P}(\tau>T)$ which is smaller than one. Of the 50000 simulated trajectories discussed below we found in all cases that $\tau>T$, so $E\left(N_{T}\right)$ must be very close to one in most situations occuring in practice. In particular, we have proved that the price of a European call option with exercise price $q>0$ and time of maturity $T$ is

$$
\pi=E\left(1_{\{\tau>T\}}\left(Z_{T}-e^{-r T} q\right)^{+}\right) .
$$

The price (4.7) can easily be calculated numerically by simulating a large number of trajectories of the process $Z$, for instance by the order 1.5 strong Taylor scheme. If $Z^{(i)}$ denotes the $i$ 'th simulation, the price is approximately equal to

$$
\hat{P}=\frac{1}{N} \sum_{i=1}^{N}\left(Z_{t}^{(i)}-e^{-r t} q\right)^{+}
$$

where all trajectories that become non-positive are discarded. In the 50000 simulations mentioned below no trajectories had to be discarded. The same method can be used to calculate the price of other European contingent claims. Direct numerical determination of the right-hand side of (4.3) is very much more complicated and time-consuming. 
We consider the special case where the daily interest rate is $r=0.0002$, the time of maturity is $T=100$, and the exercise price is $q=400$. The parameters entering the hyperbolic diffusion process model for the stock price were set to the parameter estimates obtained for the Baltica shares, (see Table 1). The instantaneous volatility in the Black-Scoles formula was set to 0.02 based on the usual quadratic variation estimator and the Baltica data. Figure 11 shows the ratio of the option price given by (4.7) to the option price given by the Black-Scholes formula as a function of the stock price at time zero. We have also included 95\%-confidence bands for the estimated curve. In this case $N=50000$.

The plot in Fig. 11 shows that the option price determined from the hyperbolic diffusion model by (4.7) is very close to the option price determined from the Black-Scholes model. This illustrates the robustness of the Black-Scholes formula to model assumptions.

Acknowledgement. The authors are grateful to Uwe Küchler, J.A. Nielsen, and T.H. Rydberg for very helpful discussions and to Lars Muus for putting the data at our disposal.

\section{References}

Arbib, M.A.: Hitting and martingale characterizations of one-dimensional diffusions. Z. Wahrscheinlichkeitstheorie verw. Gebiete 4, 232-247 (1965)

Barndorff-Nielsen, O.E.: Exponentially decreasing distributions for the logarithm of particle size. Proc. Roy. Soc. London A 353, 401-419 (1977)

Barndorff-Nielsen, O.E., Kent, J., and Sørensen, M.: Normal variance-mean mixtures and z-distributions. Int. Statist. Rev. 50, 145-159 (1982)

Bibby, B.M.: Optimal combination of martingale estimation functions for discretely observed diffusion processes. Research Report NO. 298, Department of Theoretical statistics, Institute of Mathematics, university of Aarhus, 1994

Bibby, B.M., Sørensen, M.: Martingale estimation functions for discretely observed diffusion processes. Bernoulli 1(1):17-39 (1995a)

Bibby, B.M., Sørensen, M.: On estimation for discretely observed diffusions: A review. Research Report No. 334, Department of Theoretical Statistics, University of Aarhus. Theory of stochastic processes (to appear 1995b)

Blæsild, P., Sørensen, M.K.: HYP - A computer program for analyzing data by means of the hyperbolic distribution. Research Report No. 248, Department of Theoretical statistics, Institute of Mathematics, University of Aarhus, 1992

Eberlein, E., Keller, U.: Hyperbolic distributions in finance. Bernoulli 1 (3), 281-299 (1995)

Engelbert, H.J., Schmidt, W.: On the behaviour of certain functionals of the Wiener process and applications to stochastic differential equations. In: Stochastic differential systems (Lect. Notes control Inf. Sci., No. 36, pp. 47-55) Berlin, Heidelberg, New York: Springer 1981

Engelbert, H.J., Schmidt, W.: On solutions of one-dimensional stochastic differential equations without drift. Z. Wahrscheinlichkeitstheorie verw. Gebiete 68, 287-314 (1985)

Fama, E.F.: The behaviour of stock market prices. J. Business 38, 34-105 (1965)

Florens-Zmirou, D.: On estimating the diffusion coefficient from discrete observations. J. Appl. Probab. 30, 790-804 (1993)

Godambe, V.P., Heyde, C.C.: Quasi-likelihood and optimal estimation. Int. Statist. Rev. 55, 231-244 (1987)

Hansen, A.: Complete market pricing in the Wiener filtration: A clarification of regularities. Preprint 1995 
Harrison, J.M., Pliska, S.R.: Martingales and stochastic integrals in the theory of continous trading. Stoch. Proc. Appl. 11, 215-260 (1981)

Karlin, S., Taylor, M.H.: A second course in stochastic processes. Academic Press, Orlando 1981

Kloeden, P.E., Platen, E.: Numerical solution of stochastic differential equations. Berlin, Heidelberg, New York: Springer 1992

Küchler, U., Neumann, K., Sørensen , M., Streller, A.: Stock returns and hyperbolic distributions. Discussion Paper No. 23, Sonderforschungsbereich 373, Humboldt Universität zu Berlin, 1994

Mandelbrot, B.: The variation of certain speculative prices. J. Business 36, 394-419 (1963)

Pedersen, A.R.: Uniform residuals for discretely observed diffusion processes. Research Report No. 292, Department of Theoretical Statistics, Institute of Mathematics, University of Aarhus, 1994

Rydberg, T.H.: Existence of unique equivalent martingale measures in a Markovian setting. Research Report No. 343, Department of Theoretical Statistics, University of Aarhus, 1996

Skorokhod, A.V.: Asymptotic methods in the theory of stochastic differential equations. American Mathematical Society, Providence, Rhode Island, 1989

Taylor, S.: Modelling financial time series. Chichester: John Wiley \& Sons 1986 